\title{
Opinion Mining of Thai Politics on Facebook Status Updates
}

\author{
Panida Songram, Chatklaw Jareanpon \\ POLAR Lab, Department of Computer Science, Faculty of Informatics, \\ Mahasarakham University, Mahasarakham, Thailand \\ Corresponding Author: panida.s@msu.ac.th
}

\begin{abstract}
Currently, social networking websites are widely used for communication among people. A multitude of websites are available for sharing ideas and knowledge. Facebook is the most popular social networking website in the world. Thai people widely use status updates on Facebook for all types of discussion topics: political issues, religious issue, technology, education, etc. The discussion topics express their opinion in the form of text. The opinion is very important part of making any decision. This paper is proposed to mine opinion of Thai people about the current government revolution. The opinion was extracted from the Facebook statuses updates which were written in Thai language. Two feature extraction methods were implemented. First, the traditional pre-process of text mining was used for extracting the features. Second, positive and negative words were collected to construct a sentiment lexicon to extract features. Comparative experiments were performed among Naïve Bayes, SVM (Support Vector Machines), KNN (K-Nearest-Neighbor) and decision trees. From the experimental results, they were shown that KNN gave highest accuracy when using the lexicon for feature extraction.
\end{abstract}

Keywords: Opinion mining, Sentiment analysis, Data mining, Thai politics, Facebook status updates.

\section{Introduction}

Social networking websites are widely used for communication with people around the world. Those websites are a very powerful medium for sharing ideas and knowledge. Moreover, they are used as spaces for expressing opinion. Currently, there are many social networking websites, such as Facebook, Twitter, and
LinkedIn. Facebook is the most popular social networking website. There are 900,000,000 - estimated unique monthly visitors ${ }^{(1)}$. Facebook has become a communication tool in people's daily life. People can write their perceptions on any discussion topics, such as political issues, religious issues, technology, produce, movie reviews, etc. ${ }^{(2)}$. This open style encourages people to naturally express their perceptions ${ }^{(3)}$. Therefore, the available datasets on Facebook has been exponentially increased and become very useful. Therefore, many researchers use Facebook as a source of knowledge instead of paper based surveys.

In Thailand, Facebook is the most popular social networking website. Most Thai people share and express their opinion, knowledge, and experience using short statuses update on Facebook. The status updates on Facebook is a very useful dataset for retrieving the user's knowledge or opinion on a particular topic. Facebook is a good source for finding certain information of Thai people. This paper aims to mine opinion of Thai people about the current government revolution from Facebook status updates written in Thai. First, the Facebook status updates are retrieved from the Facebook website by using Facebook Graph API. Two feature extraction methods were implemented. In the first method, Facebook status updates are pre-processed by the traditional pre-process of text mining to extract features. The second method extracts features from a lexicon which requires a dictionary of words, each of them annotated with its semantic orientation. The positive words and negative words are collected in the lexicon. Then comparative experiments are performed among Naïve Bayes ${ }^{(4)}, \mathrm{SVM}^{(5)}, \mathrm{KNN}^{(6)}$ and decision trees (7) to discover the best classifier for mining opinion of Thai politics.

The remainder of the paper is organized as follows. The related works are mentioned in section 2 . The proposed 
methodology is explained in section 3. The experimental results are shown in section 4 . Finally, conclusion is given in section 5 .

\section{Related Works}

Opinion mining or sentiment analysis was proposed to determine opinions, attitudes and emotions of humans to some topics ${ }^{(8)}$. The retrieved information from opinion mining is very useful for making decisions in many fields. For example, a government wants to know the opinion of people before enacting policies. Opinion mining becomes a popular study on social networking websites because of the fact that users are free to express their opinion through social networking function. Many researchers have tried to extract opinions in different ways: natural language processing, text analysis and computational linguistics.

Akaichi et al. ${ }^{(9,10)}$ proposed sentiment analysis on Facebook status updates by using Naïve Bayes and SVM. The purpose of the research was to identify the nature of the status update and to link them to behaviors and sentiment characteristics. The sentiment classification is performed on the 260 Facebook status updates posted by Tunisian users during the revolution. The three lexicon types were developed to give more information language of online social network. First, the acronyms' lexicon denotes the most used acronyms, such as lol, gr8, cu. Second, the emoticons' lexicon denote the most used emotion on Facebook, such as $(;),(;$, ;), etc. Third, the interjections' lexicon contains Wow, Haha, Oh dear, etc. Then, they decided the lexicons either positive or negative. Next the feature extraction was performed to transform from original textual dataset to structured dataset. In this step, the stop words removal and stemming were done to reduce search space. The text representation is the occurrence of words. The various n-gram pattern and part-of-speed tags are used as features to classify sentiment. Next, the dataset are labeled with two classes, positive and negative. $60 \%$ of pre-processed dataset is trained to create a model and $40 \%$ of pre-processed dataset is tested to evaluate the model. The n-gram patterns were experimented to evaluate with 10 -fold cross validation, i.e. unigram, bigram, trigram, a combination of unigram and bigram, a combination of unigram and trigram, a combination of bigram and trigram, a combination of unigram and bigram as well as trigram. The experimental results showed that Naïve Bayes had high accuracy when the bigram was used for feature extraction, while SVM outperformed the Naïve Bayes when the unigram was used for feature extraction.

Ortigosa et al. ${ }^{(11)}$ proposed a new method for analyzing sentiment on Facebook status updates written in Spanish. They implemented a method in SentBuk to retrieve the status updates on Facebook and classify them. The method is a combination of lexicon-based and machine learning techniques. It tokenizes a message and assigns a score to each token using a dictionary of words, emoticon and interjection. If the token is classified to positive, the score will be 1 . The score will be -1 if the token is negative, and it is assigned to 0 if the token is neutral. This research tried to prepare data using lexicon-based before using machine-learning techniques. This research said that the accuracy is strongly influenced by the context, position of words, figures and speed. The experiments were conducted by classify dataset with different four approaches, lexicon based, decision trees with lexicon-based tagging, Naïve Bayes with lexicon-based tagging, and SVM with lexicon-based tagging. From the experimental result, it was shown SVM with lexicon-based tagging gives $83.27 \%$ of accuracy for sentiment analysis on Facebook dataset when compared with a human judge.

Troussas et al. ${ }^{(12)}$ applied Naïve Bayes to classify the people's feelings to assist language learning. Moreover, they tried to compare Naïve Bayes with Rocchio and Perceptron on unigram model. In the comparison, dataset was collected aground 7,000 status updates from 90 users and manually labels as positive or negative. The dataset is randomly split to $50 \%$ for training and $50 \%$ for testing. Three algorithms are evaluated in terms of precision, recall and F-score. From the evaluation, Rocchio has the best performance than the others. It gave $75 \%$ of precision, $73 \%$ of recall and $74 \%$ of F-measure.

Shrivatava and Pant (3) developed a classifier to extract three classes, GOOD, BAD, and AVERAGE, on Facebook status updates. The Facebook puller was implemented to collect 2,000 comments from Facebook. Then the collected dataset was classified into the three classes by using a domain dictionary containing some synonyms of features. Next LIBSVM program ${ }^{(13)}$ was applied to train and test accuracy of the system. From accuracy test, it showed that the average accuracy is $70.5 \%$ on a Facebook dataset.

Jamoussi and Ameur (14) proposed sentimental orientation of Facebook comments by using linguistic approach. This paper grouped words present in corpus into two dictionaries, positive and negative dictionaries, that exploits the emotions symbols. Then the dictionary was 
used to predict either a positive or negative on Facebook comments. The dataset is collected by using Facebook API and presented in XML. Only political category pages are selected to construct the corpus, which consists of 23 Tunisian pages, 5 French pages and 4 English pages. The corpus is decomposed into two disjoint corpuses, learning corpus and test corpus. The learning corpus is divided into two subsets of comments. The first is based on comments containing emotion symbols. The second is based on the rest comments which have no emotions. The emotion symbols are used to split a comment and to identify words in either positive or negative dictionary. From the experiment results, it was shown that the proposed method has an efficiency of $80.89 \%$ and lower error rate of $18.75 \%$. Irfan et al. ${ }^{(15)}$ proposed a survey of text mining on social network websites. It studied not only the various text mining techniques but also the pre-process of text mining. In addition, the paper provides the several ideas for future research in social networking websites. For pre-process, the paper concluded that there are three basic methods, feature extraction, feature selection and document representation. The feature extraction is divided to morphological analysis, syntactical analysis, and semantic analysis. The morphological analysis deals with word representation that consist of stop word removal, stemming. The syntactical analysis deals with the grammatical structure of language. The syntactical analysis deals with part of speed tagging and parsing. For feature selection, it selects only important features to remove redundant information and then make a reduced cost. The important features are mostly selected by scoring the words. The score of the words may be calculated from frequency, latent semantic indexing, and random mapping. Then they are represented as a vector space model. For text classification, the paper grouped text classification approaches into machine learning based approach, ontology based approach, and hybrid approach. From the survey, they conclude that the hybrid approach has better performance than applying a single method. For future research, the paper suggests that interpreter of emotions on social networks is a challenging issue because textual data on social network becomes very large, noisy, dynamic, unstructured language. For sentiment classification approaches, they are grouped into 3 groups. The first group is lexicon-based approaches that classification by using words of lexicon. The second group is machine learning approaches, such as SVM, Naïve Bayes, Rocchio and so on. The third group is hybrid approaches that combine the lexicon-based approaches and machine learning approaches together. The hybrid approaches have better performance than the others.

\section{The Proposed Methodology}

Fig. 1 shows the overall process of the proposed methods. First, 467 status updates written in Thai are collected from Facebook during 22 May 2014 until 17 July 2014 by using Facebook Graph API ${ }^{(16)}$. The status updates are about Thai people's opinion of the current in country revolution. The status updates were manually identified whether positive or negative by human and divided into 259 positive and 208 negative status updates. The 102 positive and 101 negative words are collected from Facebook to construct a sentiment lexicon. After collecting the dataset and lexicon, the following steps will be performed.

Step 1 Cleaning: Before the pre-processing step, the dataset has to be cleaned because the writing on social networking websites is normally not correct. Therefore, some words in a dataset are changed to the correct form to improve efficiency and effectiveness.

Step 2 Feature Extraction: Since the dataset is text data that is unstructured, it has to be transformed to structural data that is a vector space model. The vectors are used to build a classifier. In this paper, the vectors are constructed from two methods as follows.

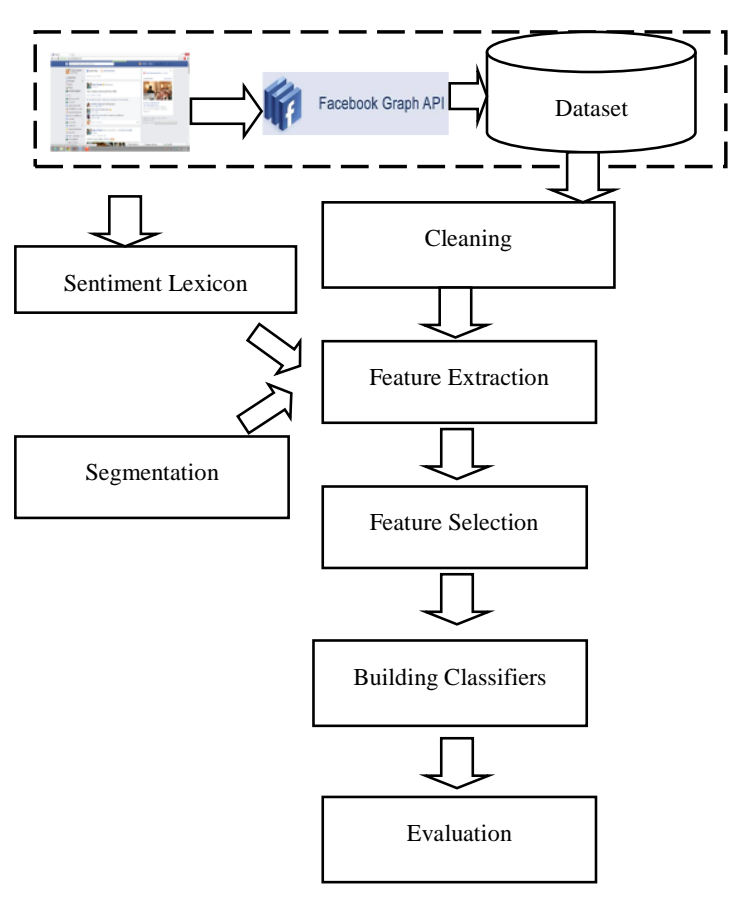

Fig. 1. The proposed methodology 
The first method: The vectors are constructed from using the traditional pre-processing steps of text mining. First, Facebook status updates are segmented by using the longest matching approach ${ }^{(17)}$, which is dictionary-based approach, to find a collection of features. Each feature is a longest meaning Thai word. Second, stop words, such as prepositions, pronouns, numbers, are removed to improve the efficiency because a large number of the stop words always appear in status updates and may reduce the efficiency of the text classification. Third, features having similar meaning are matched into a feature. It is used to reduce the large number of features. In this step, the matching is done by using a dictionary that is constructed from a collection of words and the similar meaning of the words. After that a status update is transformed to a vector. The vector consists of the set of weights of features as shown in Table 1. The weight of features is calculated by using Boolean weighting as shown in equation (1), where $w_{k i}$ is the weight of feature $i$ in status update $k$ and $f_{i}$ is the number of occurrences of the feature. The weight of feature is 1 if the feature occurs in the status update. Otherwise, the weight of feature is 0 .

The second method: The vectors are constructed from the lexicon that is created from a collection of positive and negative words. The collection of words is used as a set of features. For each status update, if a positive or negative word is found in the status update, the weighting is set to 1 ; otherwise, the weighting is set to 0 as shown in equation (1).

Table 1. Example Vector

\begin{tabular}{|l|c|c|c|c|}
\hline Document & Feature $_{1}$ & Feature $_{2}$ & $\ldots$ & Feature $_{\mathrm{i}}$ \\
\hline$d_{1}$ & 1 & 0 & $\ldots$ & 0 \\
\hline$d_{2}$ & 0 & 1 & $\ldots$ & 1 \\
\hline$\ldots$ & $\ldots$ & $\ldots$ & $\ldots$ & $\ldots$ \\
\hline$d_{\mathrm{k}}$ & 1 & 1 & $\ldots$ & 1 \\
\hline
\end{tabular}

$$
w_{k i}=\left\{\begin{array}{l}
1 \text { iff } f_{i}>0 \\
0 \text { otherwise }
\end{array}\right.
$$

$$
\text { Accuracy }=\frac{(t p+t n)}{(t p+t n+f p+f n)}
$$

Step 3 Feature Selection: After extracting features, the feature selection is performed to eliminate insignificant features. A feature is removed if the occurrence of feature is less than a threshold. The threshold in this paper is the number of status updates. Four thresholds are set in the experiments: 4, 8 and 12 .

Step 4 Building Classifiers: To evaluate the extraction method, the pre-processed datasets are divided to training set and testing set using 10 -fold cross validation. The training set is used to create classifiers based on four methods, Naïve Bayes, SVM, KNN and decision trees.

Step 5 Evaluation: The classifiers are evaluated on a testing set. The accuracy of the prediction is used to evaluate the effectiveness of classifiers. The accuracy can be computed in equation (2), where $t p$ is the number of correct predictions that an instance is positive, th is the number of correct predictions that an instance is negative, $f p$ is the number of incorrect predictions that an instance is positive and $f n$ is the number of incorrect of predictions that an instance negative.

\section{Experimental Results}

In this paper, all experiments are performed to analyze the accuracy of opinion mining. All experiments are run on a $1.90 \mathrm{GHz}$ Core ${ }^{\mathrm{TM}} \mathrm{i} 3 \mathrm{PC}$ with $4 \mathrm{G}$ of main memory, running on Microsoft Windows 7. All algorithms are implemented on Matlab. Datasets are generated in different ways. The first method tried to extract features from all the meaning words that appeared in the messages collected from Facebook status updates. The Facebook status updates were pre-processed by using the pre-process steps of text mining. The resulting dataset is called Data1. The second method extracted features from the lexicon that are created from a collection of positive and negative words. Data2 is the dataset that is pre-processed from the second method. Data2-4, Data2-8, Data2-12 passed the feature selection step using the threshold values, 4,8 and 12 , respectively. The characteristic of all datasets is shown in Table 2.

Table 2. The characteristics of datasets

\begin{tabular}{|l|l|l|}
\hline \multicolumn{1}{|c|}{ Dataset } & \multicolumn{1}{|c|}{ \#Features } & \multicolumn{1}{c|}{ \#Status updates } \\
\hline Data1 & 5022 & 467 \\
\hline Data2 & 203 & 467 \\
\hline Data2-4 & 97 & 467 \\
\hline Data2-8 & 50 & 467 \\
\hline Data2-12 & 30 & 467 \\
\hline
\end{tabular}


Table 3. The accuracy on Data1

\begin{tabular}{|l|l|}
\hline \multicolumn{1}{|c|}{ Classifier } & \multicolumn{1}{c|}{ Accuracy (\%) } \\
\hline Decision Trees & 29.08 \\
\hline Naïve Bayes & 55.59 \\
\hline KNN & 54.93 \\
\hline SVM & 53.64 \\
\hline
\end{tabular}

Table 4. The accuracy on Data2

\begin{tabular}{|l|l|}
\hline \multicolumn{1}{|c|}{ Classifiers } & \multicolumn{1}{c|}{ Accuracy (\%) } \\
\hline Decision Trees & 22.89 \\
\hline Naïve Bayes & 51.44 \\
\hline KNN & 62.37 \\
\hline SVM & 59.31 \\
\hline
\end{tabular}

Table 5. The accuracy of KNN

\begin{tabular}{|l|l|}
\hline \multicolumn{1}{|c|}{ Dataset } & \multicolumn{1}{c|}{ Accuracy (\%) } \\
\hline Data2 & 62.37 \\
\hline Data2-4 & 62.77 \\
\hline Data2-8 & 63.58 \\
\hline Data2-12 & 58.92 \\
\hline
\end{tabular}

Table 3 shows the accuracies of Naïve Bayes, SVM, KNN and decision trees on Data1. The Naïve Bayes gives the best accuracy when comparing to SVM, KNN and decision trees. The accuracy of decision trees is less than $50 \%$ and the accuracy of SVM, Naïve Bayes and KNN is still low because of the many different features appearing in the dataset and a lot of zero values in the dataset make the effective classifier low.

Table 4 shows the accuracies of Naïve Bayes, SVM, $\mathrm{KNN}$ and decision trees on Data2. From the table, it is shown that the accuracies of KNN and SVM are increased and the accuracy of $\mathrm{KNN}$ is increased till $62.37 \%$ while the accuracies of decision trees and Naïve Bayes are down. Therefore, KNN is the best approach for analysis of Thai people's opinion when using the lexicon for feature extraction.

Table 5 shows the accuracies of $\mathrm{KNN}$ on four datasets, Data2, Data2-4, Data2-8 and Data2-12. From the table, it is shown that the accuracies are not too much different. The best accuracy is $63.58 \%$ on Data2-8.

From Table 3, Table 4 and Table 5, the best method for mining Thai people's opinion about the revolution is KNN when comparing to Naïve Bayes, SVM and decision trees. Extracting features from the lexicon is better than using all the meaning words that appeared in the Facebook status updates.

\section{Conclusion}

This research proposed to mine opinion of Thai politics from Facebook status updates. It tried to extract features from different methods. The first method is that features are extracted from the status updates using traditional pre-process of text mining. The second method is to extract features from the lexicon which requires a collection of positive and negative words. Then Naïve Bayes, SVM, KNN and decision trees are evaluated on the pre-processed datasets in terms of accuracy. Experimental results show that KNN gives the highest accuracy when using positive and negative words for extraction. It provides $63.58 \%$ of accuracy for mining opinions of Thai politics. To improve the accuracy in the future works, sarcasm detection of opinions about the politics is a challenging issue. Moreover, adding acronyms' lexicon, emoticons' lexicon, and interjections' lexicon may be improve the accuracy.

\section{Acknowledgment}

This research was funded by Faculty of Informatics, Mahasarakham University.

\section{References}

(1) eBizMBA : "Top 15 Most Popular Social Networking Sites" | April 2014, Retrieved 8 April 2014, from http://www.ebizmba.com/articles/ social-networking-websites. Alexander Pak and Patrick Paroubek : "Twitter as a Corpus for Sentiment Analysis and Opinion Mining", In Proceedings of the Seventh conference on International Language Resources and Evaluation (LREC'10), Valletta, Malta: European Language Resources Association (ELRA), pp. 35-39, 2010

(3) Akash Shrivatava and Bhasket Pant : "Opinion Extraction and Classification of Real Time Facebook", Global Journal of Computer Science and Technology, Vol.12, Issue 8, 2012

(4) Nir Friedman, Dan Geiger, and Moises Goldszmidt : "Bayesian Network Classifiers. Machine Learning", Vol. 29, Issue 2-4, pp. 131-163, 1997

(5) Corinna Cortes and Vladimir Vapnik : "Support-Vector Networks", Mach Learn, Vol. 20, pp. 273-297, 1995

(6) Gongde Guo, Hui Wang, David Bell, Yaxin Bi, and Kieran Greer : "KNN Model-Based Approach in 
Classification", On The Move to Meaningful Internet Systems 2003, CoopIS, DOA, and ODBASE Lecture Notes in Computer Science Vol. 2888, pp 986-996, 2003

(7) J. R. Quinlan : "Introduction of Decision Trees", Machine Learning, Vol.1, pp. 81-106, 1986

(8) Bing Liu and Lei Zhang : "A Survey of Opinion Mining and Sentiment Analysis", Mining Text Data, pp. 415-463, 2012

(9) Jalel Akaichi, Zeineb Dhouioui, Maria Jose Lopez-Huertas Perez : "Text Mining Facebook Status Updates for Sentiment Classification", In Proceeding of the 17th International Conference on System Theory, Control and Computing (ICSTCC), Sinaia, pp. 640-645, 2013

(10) Jalel Akaichi : 'Social Networks' Facebook' Statutes Updates Mining for Sentiment Classification", In Proceedings of the 2013 International Conference on Social Computing, Washington, DC; United States, pp.886-891, 2013

(11) Alvaro Ortigosa, Jose M. Martín, and Rosa M. Carro : "Sentiment Analysis in Facebook and Its Application to E-learning", Compuers in Human Behavior, Vol. 31, pp. 527-541, 2014

(12) Cristos Troussas, Maria Virvou, Kurt Junshean Espinosa, Kevin Llaguno, and Jaime Caro : "Sentiment Analysis of Facebook Status Using Naive Bayes Classifier for Language Learning", In Proceeding of 4th International Conference on Information, Intelligence, Systems and Application. Piraeus, pp. 1 - 6, 2013

(13) Chih-Chung Chang and Chih-Jen Lin : "LIBSVM-A Library for Support Vector Machines", Journal ACM Transactions on Intelligent Systems and Technology, Vol. 2. Issue 3, No. 27, 2011.

(14) Salma Jamoussi, Hanen Ameur : "Dynamic Construction of Dictionaries for Sentiment Classification", In Proceeding of the 3rd International Conference on Cloud and Green Computing (CGC), pp. 418-425, 2013.

(15) Rizwana Irfan, Christine King, Daniel Drages, et al : "A Survey on Text Mining in Social Networks", The Knowledge Engineering Review, pp. 1-24, 2004

(16) Anonymous. The Graph API, Retrieved 23 August 2014, from https://developers.facebook.com/docs/ graph-api. cite

(17) Surapant Meknavin, Paisarn Charoenpornsawat, and Boonserm Kijsirikul : "Feature-based Thai Word Segmentation", In Computer Engineering, . Bangkok, Chulalongkorn University, 1999. 